\title{
Beam loading effects and microwave instability in the booster ring of a high intensity heavy-ion accelerator facility
}

\author{
F. C. Cai $\oplus^{1,2,3}$ J. C. Yang $\oplus^{1,3^{*}}$ J. W. Xia, ${ }^{1,2,3}$ G. D. Shen, ${ }^{1,3}$ J. Liu $\odot,{ }^{1,3}$ L. J. Mao, ${ }^{1,3}$ D. Y. Yin, ${ }^{1,3}$ \\ Y. Cong, ${ }^{1,3}$ S. Ruan, ${ }^{1,3}$ G. Wang, ${ }^{1,2,3}$ L. P. Yao, ${ }^{1,2,3}$ and X. Q. Chen ${ }^{1,2,3}$ \\ ${ }^{1}$ Institute of Modern Physics, Chinese Academy of Sciences, Lanzhou 730000, China \\ ${ }^{2}$ University of Chinese Academy of Sciences, Beijing 100049, China \\ ${ }^{3}$ Huizhou Research Center of Ion Science, Huizhou 516003, China
}

(Received 20 October 2019; accepted 28 May 2020; published 15 June 2020)

\begin{abstract}
Transient beam loading effects and microwave instability are very important in ion synchrotrons. A simulation code longitudinal particle tracking code (LPTC) based on multiparticle tracking is developed to simulate the beam loading effects and the longitudinal beam instabilities in the booster ring (BRing) of the High Intensity heavy-ion Accelerator Facility (HIAF). A process of beam capture, acceleration, debunching, and the second capture is simulated with a reference ion beam of ${ }^{78} \mathrm{Kr}^{19+}$. The simulation results show that the beam loading effects have major contribution to the beam behavior during the debunching stage and the second capture stage. A feed-forward system is suggested to compensate the beam loading effects, and the main parameters of the feed-forward system are given. The microwave instability of coasting ${ }^{78} \mathrm{Kr}^{19+}$ beams is observed from the simulation results. The threshold of the microwave instability is also given. The development of self-bunching and methods to suppress the microwave instability in the BRing are discussed.
\end{abstract}

DOI: 10.1103/PhysRevAccelBeams.23.064402

\section{INTRODUCTION}

The High Intensity heavy-ion Accelerator Facility (HIAF) was proposed by Institute of Modern Physics (IMP) of the Chinese Academy of Sciences (CAS) to provide high intensity heavy ion beams for nuclear physics, atomic physics, and other applications [1]. The booster ring (BRing) is a key component of the HIAF. Ions from the injector Ion Linac (iLinac) will be accumulated in the BRing by the two-plane painting injection scheme [2]. Beam intensity up to $3 \times 10^{11}$ particles per pulse (ppp) can be obtained in the BRing after injection. A coasting beam with RMS momentum spread of 0.00067 is formed. Then the coasting beam will be captured adiabatically into several bunches and accelerated to extraction energy. Two different extraction operation modes, fast extraction and slow extraction, are designed in the BRing [3]. For the fast extraction, the bunches should be debunched and then captured into only one bunch in the ring. For the slow extraction, the bunches

\footnotetext{
Corresponding author. yangjch@impcas.ac.cn

Published by the American Physical Society under the terms of the Creative Commons Attribution 4.0 International license. Further distribution of this work must maintain attribution to the author(s) and the published article's title, journal citation, and DOI.
}

need to be debunched as a coasting beam in the ring. The main parameters of the BRing and its rf system are shown in Table I.

Several magnetic alloy (MA) loaded cavities [4] will be used for beam capture, acceleration, and debunching in the BRing. MA loaded cavities have been employed in many accelerator facilities, such as JPARC-RCS [5,6], CERN-PSB [7], GSI-SIS18 [8], and KEK-AGS [9], since they can provide high acceleration field gradient and cover wide frequency region. The quality factor ( $Q$ value) of the BRing MA loaded cavities is set at 0.45 to cover a wide frequency range of $0.3-2 \mathrm{MHz}$. The magnitude of the longitudinal impedance $(|Z|)$ of the BRing MA loaded cavities are shown in Fig. 1. The frequency sweeps of the first 6 harmonics of 3 typical beams, which will be used for analysing the bandwidth of the feed-forward system later in this paper, are also shown in Fig. 1.

MA loaded cavities can cause heavy beam loading effects [10]. Beam loading effects result from the interaction between the beams and rf cavities. The total voltage in the cavities is the superposition of the programmed $\mathrm{rf}$ voltage and the beam-induced wake voltage. The decay time of wake voltage is

$$
T_{d}=\frac{2 Q}{h \omega_{0}}=\frac{Q}{\pi h} T_{0}
$$


TABLE I. Parameters of the BRing and its rf system.

\begin{tabular}{lc}
\hline \hline Parameter & Value \\
\hline Circumference $C$ & $569.1 \mathrm{~m}$ \\
Transition energy $\gamma_{t}$ & 7.64585 \\
Momentum spread acceptance & \pm 0.005 \\
Total shunt impedance $R_{s}$ & $3.4 \mathrm{k} \Omega$ \\
$Q$ value & 0.45 \\
Resonance frequency $f_{r}$ & $1.5 \mathrm{MHz}$ \\
Maximum programmed rf voltage $V_{\text {rf,max }}$ & $240 \mathrm{kV}$ \\
\hline \hline
\end{tabular}

where $h$ is harmonic number, $\omega_{0}$ is the angular revolution frequency of the beam, and $T_{0}$ is the revolution period of the beam. The decay time is about $0.14 T_{0} / h$ in the BRing, which means only transient beam loading effects need to be concerned. Compensation of the wake voltage is the most important method to reduce the transient beam loading effects. In the BRing, beam manipulation and beam states are complicated, which means it is of importance to study whether the beam loading effects are severe at each stage in a whole operation process.

In the presence of MA loaded cavities, it is also important to study the microwave instability [11] of coasting beams with high beam intensity and small momentum spread. The microwave instability, manifested as the development of self-bunching, can lead to the momentum spread growth of the coasting beams. For the slow extraction in the BRing, coasting beams with small momentum spread are required. Thus the microwave instability of coasting beams is an important issue in the BRing.

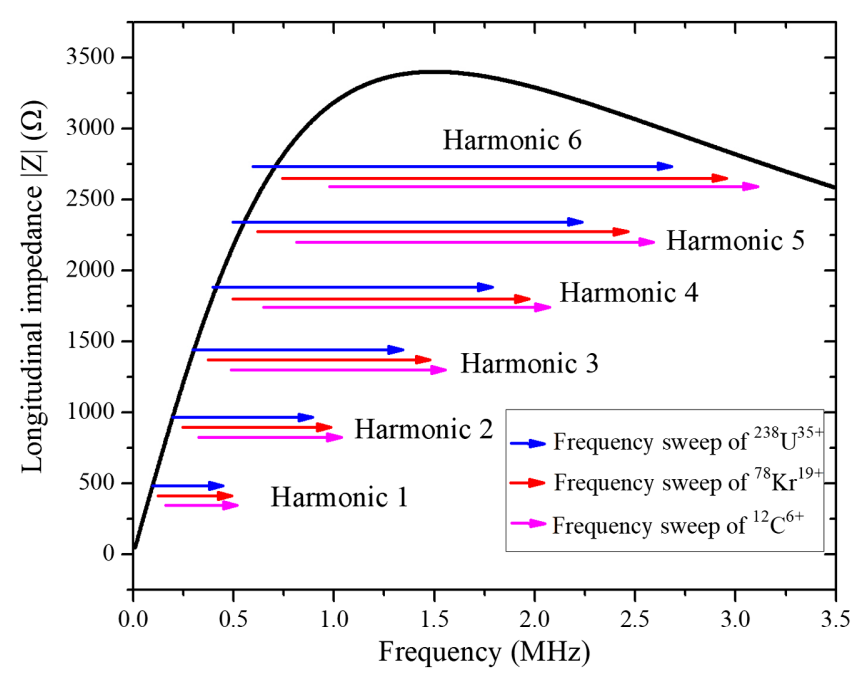

FIG. 1. The magnitude of the longitudinal impedance $(|Z|)$ of the cavities and the frequency sweeps of the first 6 harmonics of 3 typical beams: ${ }^{238} \mathrm{U}^{35+},{ }^{78} \mathrm{Kr}^{19+}$, and ${ }^{12} \mathrm{C}^{6+}$. The frequency sweeps will be used for analyzing the bandwidth of the feedforward system later in this paper.
This paper is organized as follows. In Sec. II, the development of a simulation code longitudinal particle tracking code (LPTC) is presented. In Sec. III A, the simulation results of the beam loading effects of the ${ }^{78} \mathrm{Kr}^{19+}$ beam in the BRing in a whole operation process are presented. Compensation of the beam loading effects is described, and the main parameters of a feed-forward system proposed for the BRing are given. In Sec. III B, the microwave instability of coasting beams in the BRing is studied. The threshold of the microwave instability is given. The development of self-bunching and methods to suppress the microwave instability in the BRing are discussed. Finally, the conclusions of this paper are given in Sec. IV.

\section{SIMULATION CODE DEVELOPMENT}

Particle tracking codes are powerful tools to study the behavior of beams in phase space. Some codes have been developed so far, such as ESME [12] and BLonD [13]. To study the longitudinal beam dynamics with complicated beam manipulation and multiple beam states in the BRing, the code LPTC is developed.

\section{A. Development of LPTC}

\section{Coordinates}

The coordinates adopted in the LPTC are energy difference $\Delta E[\mathrm{MeV} /$ nucleon] from synchronous energy and azimuth angle $\theta$ [rad]. The azimuth angle has the range of $[-\pi, \pi)$ and is related to the synchronous phase $\phi_{s}$ by

$$
\phi=\phi_{s}-h \theta
$$

where $\phi$ is the phase of the particle. In this coordinate system, particles move in the direction that $\theta$ increases, and the synchronous particle will always get the energy increase at $\theta=0$.

\section{Difference equations}

The single particle difference equations, which are used for tracking particles turn by turn with coordinates $\left(\theta, \Delta E / \omega_{0}\right)$, are

$$
\begin{aligned}
\Delta E_{n+1}= & \frac{\beta_{s, n+1}}{\beta_{s, n}}\left(\Delta E_{n}+D e\left\{V_{\mathrm{rf}}\left[\sin \left(\phi_{s}-h_{\mathrm{rf}} \theta_{n}\right)-\sin \phi_{s}\right]\right.\right. \\
& \left.\left.+V_{\text {wake }}\left(n, \theta_{n}\right)+V_{\text {feed }}\left(n, \theta_{n}\right)\right\}\right), \\
\theta_{n+1}= & \left(\theta_{n}-\frac{2 \pi \eta}{\beta_{s, n+1}^{2} E_{n+1}} \Delta E_{n+1}+\pi\right)_{\bmod (2 \pi)}-\pi,
\end{aligned}
$$

where the suffix $s$ represents synchronous particle, $n$ represents nth turn, $\beta$ is the ratio of the velocity of the 
synchronous particle to the speed of light, $D$ is the chargeto-mass ratio of the particles, $V_{\mathrm{rf}}$ is the programmed $\mathrm{rf}$ voltage, $h_{\mathrm{rf}}$ is the harmonic number of the rf, $V_{\text {wake }}\left(n, \theta_{n}\right)$ is the wake voltage, $V_{\text {feed }}\left(n, \theta_{n}\right)$ is the compensation voltage, $E$ is the total energy per nucleon of the particles, and $\eta$ is the phase slip factor. Since the velocity of heavy ions increases a lot during the acceleration, the part $\beta_{s, n+1} / \beta_{s, n}$ is not neglectable. Since the momentum spread $\delta$, related with energy spread $\Delta E / E$ by $\delta=\frac{\Delta E}{E} \frac{1}{\beta^{2}}$, is widely used in theoretical analysis, the $\theta-\delta$ phase space can be chosen as the output of the LPTC.

\section{Wake voltage calculation}

The wake voltage is calculated in time domain. The cavity is modeled as an RLC parallel circuit [14] of which the impedance is written as

$$
Z(\omega)=\frac{R_{s}}{1+i Q\left(\omega_{r} / \omega-\omega / \omega_{r}\right)},
$$

where $\omega$ is the angular frequency, and $\omega_{r}$ is the angular resonant frequency of the cavity. The wake function is written as [14]

$W_{0}^{\prime}(\theta)=\frac{\omega_{r} R_{s}}{Q} e^{\alpha \theta / \omega_{0}}\left[\cos \left(\frac{\bar{\omega}}{\omega_{0}} \theta\right)+\frac{\alpha}{\overline{\bar{\omega}}} \sin \left(\frac{\bar{\omega}}{\omega_{0}} \theta\right)\right]$

for $\theta<0$, where $\alpha=\omega_{r} / 2 Q$ is the decay rate, and $\bar{\omega}=\sqrt{\left|\omega_{r}^{2}-\alpha^{2}\right|}$ is the shifted resonant angular frequency. For $\theta>0, W_{0}^{\prime}(\theta)=0$. For $\theta=0$, according to the fundamental theorem of beam loading [15], the wake function is written as

$$
W_{0}^{\prime}(\theta)=\frac{\omega_{r} R_{s}}{2 Q}
$$

The wake function given by Eq. (6) is only suitable for cavities with $Q \geq 0.5$. If the $Q$ value is smaller than 0.5, which is possible for an MA loaded cavity, the cosine and sine functions in Eq. (6) should be replaced by hyperbolic cosine and hyperbolic sine functions.

The wake voltage seen by a test point charge at position $\theta$ is calculated by

$$
V_{\text {wake }}(\theta)=\int_{\theta}^{\infty} q \rho\left(\theta^{\prime}\right) W_{0}^{\prime}\left(\theta-\theta^{\prime}\right) d \theta^{\prime},
$$

where $q$ is the total charge ahead of the test charge, and $\rho\left(\theta^{\prime}\right)$ is the normalized charge distribution of the charge $q$.

In the code, hundreds of slices are cut along the ring. All particles in one slice are seen as a "supermacro" particle located at the center of the slice, and each of them generates the same wake potential. The wake voltage experienced by the particles in slice $j$ is calculated by summing all the wake voltage induced by the supermacro particles ahead of the particles and the wake voltage induced in the previous turns. Thus the wake voltage $V_{\text {wake }}\left(n, \theta_{n}\right)$ in Eq. (3) is

$V_{\text {wake }}\left(n, \theta_{n}\right)=\Sigma_{0}^{K} \Sigma_{0}^{M-1} q_{n-k, m} W_{0}^{\prime}\left[-2 \pi\left(k+\frac{m-j}{M}\right)\right]$,

where $2 \pi j / M-\pi \leq \theta_{n}<2 \pi(j+1) / M-\pi, \quad m$ is the number of slice, $n$ is the number of turn, $q_{n-k, m}$ is the total charge in mth slice of $(n-k)$ th turn, $K$ is the upper limit of the turns to calculate the wake voltage, and $M$ is the number of slices along the ring.

\section{Wake compensation calculation}

To compensate the beam loading effects, the wake voltage $V\left(n, h \omega_{0}\right)$ in frequency is calculated by

$$
V_{\text {wake }}\left(n, h \omega_{0}\right)=Z\left(h \omega_{0}\right) \cdot I\left(n, h \omega_{0}\right),
$$

where $Z\left(h \omega_{0}\right)$ is the impedance of the cavity at angular frequency $\omega=h \omega_{0}$, and $I\left(n, h \omega_{0}\right)$ is the harmonic current at harmonic $h$. The harmonic current is obtained from the beam distribution by means of the fast Fourier transform (FFT). Since the feed-forward system usually has a delay, the wake is calculated with the beam current of $\Delta n$ turns earlier, which means the harmonic current $I\left(n, h \omega_{0}\right)$ should be replaced by $I\left(n-\Delta n, h \omega_{0}\right)$ :

$$
V_{\text {wake }}\left(n, h \omega_{0}\right) \approx Z\left(h \omega_{0}\right) \cdot I\left(n-\Delta n, h \omega_{0}\right) .
$$

The compensation voltage $V_{\text {feed }}\left(n, \theta_{n}\right)$ in Eq. (3) is then produced in time domain through inverse FFT as

$V_{\text {feed }}\left(n, \theta_{n}\right)=-\Sigma_{h \in A} \bar{V}_{\text {wake }}\left(n, h \omega_{0}\right) \sin \left(h \theta_{n}+\phi_{h}\right)$,

where $A$ is an array that contains the harmonic numbers at which the wake should be compensated, $\bar{V}_{\text {wake }}(n, h \omega)$ is the amplitude of $V_{\text {wake }}\left(n, h \omega_{0}\right)$, and $\phi_{h}$ is the phase difference of $V_{\text {wake }}\left(n, h \omega_{0}\right)$ relative to the synchronous phase.

\section{B. Characteristics of LPTC}

The code LPTC has many characteristics which insure that the LPTC can be used to perform longitudinal beam dynamics simulations with wake and wake compensation for heavy ion accelerators such as the BRing.

First, the normalized phase space keeps constant during the acceleration, and the calculation of wake function is amended for $Q<0.5$. Thus the LPTC is capable of 
simulating the longitudinal dynamics of heavy ion beams in accelerators with low $Q$ value MA loaded cavities.

Second, since the $Q$ value of MA loaded cavities is small, wake voltage damps quickly. The number of slices along the ring should be large enough so that the wake voltage is approximately invariant inside one slice. However, the computation time of wake voltage is proportional to the square of the number of slices. To reduce computation time, the ring is automatically cut into slices according to the duty ratio of the beams, and the wake voltage is calculated only in the slices containing particles.

Third, if the manipulation of the beams is complicated, each longitudinal manipulation stage can be treated separately, which is very important to study the beam loading effects at each stage. For example, since harmonic currents change a lot after rf harmonic number changes, the influence of each harmonic current on the beams should be studied separately. In the LPTC, if necessary, it is convenient to choose which harmonic current should be compensated by setting different arrays of harmonic number at different stages.

\section{Benchmarks of LPTC}

To ensure that the LPTC is reliable, some benchmarks with existing simulation codes and theoretical calculations have been done.

The BLonD code, which is developed at CERN to simulate the longitudinal dynamics in synchrotrons, has been proven to be well developed [16]. Simulations have been performed with the BLonD and the LPTC. Since the BLonD is only applicable for the case with $Q>0.5$, the $Q$ value is set at 0.6 . The Fourier transform of bunch length and bunch center oscillations in the presence of wake are shown in Fig. 2. The angular frequency $\omega$ is normalized by the angular revolution frequency $\omega_{0}$. The peaks are related to the synchronous oscillation. The differences between the magnitudes of each peak are less than $0.225 \mathrm{~dB}$, and the differences between the centers of each peak are less than $2 \times 10^{-5} \omega_{0}$. The results of LPTC simulations agree very well with the results of BLonD simulations.

The potential-well distortion, which is caused by the wake voltage, can lead to the change of bunch distribution. The linear beam density of an electron bunch with Gaussian distribution in energy spread can be derived from the Haissinski equation [11]. The Haissinski equation can be solved analytically with a pure resistive impedance $R_{\mathrm{pr}}$, and the solution gives the linear distribution as

$$
\lambda(\theta)=\frac{\sqrt{2 / \pi} e^{-\theta^{2} /\left(2 \sigma_{\theta}^{2}\right)}}{\xi \sigma_{\theta}\left[\operatorname{coth}\left(\xi q_{b} / 2\right)-\operatorname{erf}\left(\theta /\left(\sqrt{2} \sigma_{\theta}\right)\right)\right]},
$$

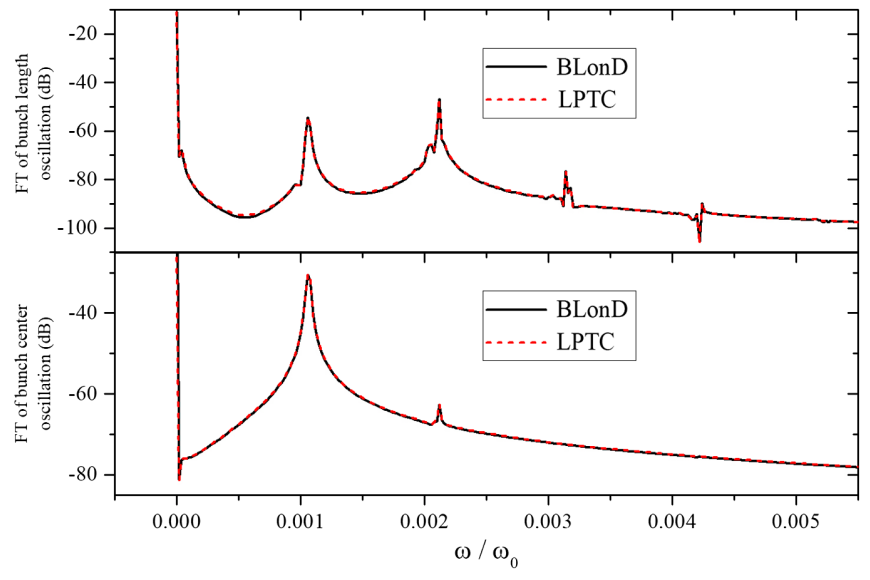

FIG. 2. Fourier Transform (FT) of bunch length (top) and bunch center (bottom) oscillations. The angular frequency is normalized by the angular revolution frequency $\omega_{0}$.

where $\sigma_{\theta}$ is the RMS bunch length, $\operatorname{erf}(x)$ is the error function, $q_{b}$ is the total charge of the bunch, and

$$
\xi=\frac{e^{2} \beta^{2} E R_{\mathrm{pr}}}{\eta T_{0} \sigma_{E}^{2}}
$$

where $\sigma_{E}$ is the RMS energy spread determined by synchrotron radiation. The Haissinski equation can also be used to describe the potential-well distortion of a heavy ion bunch with Gaussian distribution in energy spread. Simulations, in which the synchrotron radiation is ignored, have been performed with heavy ion beams. In the simulations, the initial bunches with Gaussian distributions in both energy spread and linear density are generated to match the buckets created by rf voltage. In each simulation, the impedance $R_{\mathrm{pr}}$ slowly increases from 0 to its maximum value so that the bunches are distorted adiabatically. The bunch distributions according to the Haissinski equation and LPTC simulation results are shown in Fig. 3. The LPTC simulation results agree with the theoretical calculations under different beam intensities.

With many benchmarks including the two benchmarks above, the LPTC is proved to be reliable and can be used for the study of longitudinal beam dynamics in the BRing.

\section{SIMULATIONS OF LONGITUDINAL BEAM DYNAMICS}

The simulations are performed with the ${ }^{78} \mathrm{Kr}^{19+}$ beam because the charge intensity of the ${ }^{78} \mathrm{Kr}^{19+}$ beam is the highest among all the heavy ion beams in the BRing. After the injection, the density distribution of the coasting beam in the longitudinal phase space is assumed to be uniform distribution on the $\theta$ axis and Gaussian 


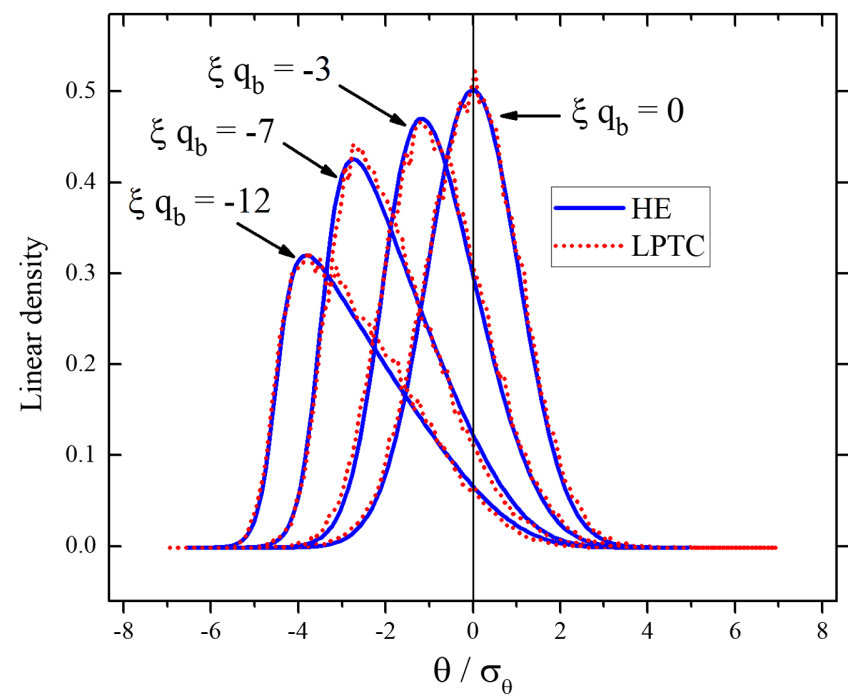

FIG. 3. Bunch distributions according to the Haissinski equation (HE) and LPTC simulation results. With different beam intensities, the values of $\xi \cdot q_{b}$ are calculated and shown in the figure. The linear density is normalized to $\sqrt{\pi / 2} \sigma_{\theta} / \omega_{0}$ when integrated over $\theta$.

distribution on the $\delta$ axis. The beam parameters are listed in Table II.

A typical programmed rf voltage for the ${ }^{78} \mathrm{Kr}^{19+}$ beam is shown in Fig. 4. The synchronous phase, which is calculated from the voltage program and the ramp of the magnetic field, is also shown. There are four stages as shown in Fig. 4. At the acceleration stage, the maximum ramping rate of bending magnetic field $(\dot{B})$ is $12 \mathrm{~T} / \mathrm{s}$. In the fast extraction mode, the extraction starts at the end of the second capture stage. In the slow extraction mode, only the first three stages are needed, and the slow extraction starts at the end of the debunching stage. The synchronous energy of the beam, which is a reference to each stage, is included in Fig. 4 as well as some other figures in this paper.

In the simulations performed with the LPTC, two beam states are of concern here: the bunched beam in a whole operation process and the coasting beam for the slow extraction.

TABLE II. Beam parameters for simulation.

\begin{tabular}{lc}
\hline \hline Parameter & Value \\
\hline Ion species & ${ }^{78} \mathrm{Kr}^{19+}$ \\
Injection energy $E_{\text {inj }}$ & $27 \mathrm{MeV} /$ nucleon \\
Extraction energy $E_{\text {ext }}$ & $1700 \mathrm{MeV} /$ nucleon \\
Harmonic number $h$ & 3,1 \\
Momentum spread (RMS) at injection $\delta$ & 0.00067 \\
Beam intensity & $3 \times 10^{11} \mathrm{ppp}$ \\
Revolution frequency $f_{0}$ & $0.12-0.49 \mathrm{MHz}$ \\
\hline \hline
\end{tabular}

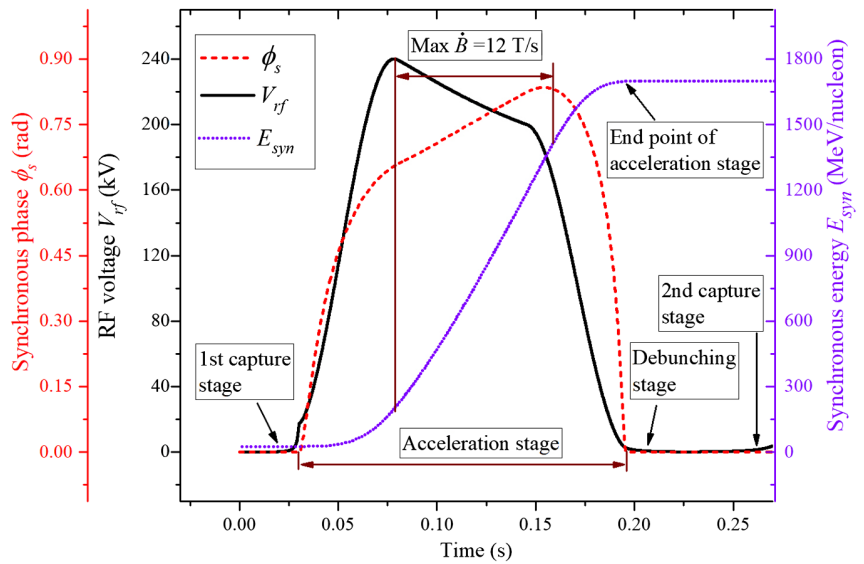

FIG. 4. Programmed rf voltage for the ${ }^{78} \mathrm{Kr}^{19+}$ beam. The synchronous phase is calculated from the voltage program and the ramp of magnetic field. There are four stages in a whole operation process: the first capture stage, acceleration stage, debunching stage, and the second capture stage. The synchronous energy of the beam is included as a reference to each stage.

\section{A. Beam loading effects}

The effective voltage seen by the beam is the superposition of the programmed rf voltage and the wake voltage. The wake voltage will induce a bucket distortion. The ratio of the wake voltage to the programmed rf voltage is an important factor to determine the severity of the bucket distortion.

The maximum wake voltage and the ratio of the maximum wake voltage to the programmed rf voltage during a whole operation process are shown in Fig. 5. The largest wake voltage reaches $8.2 \mathrm{kV}$ at the end of the acceleration with the maximum $\dot{B}$ while the corresponding

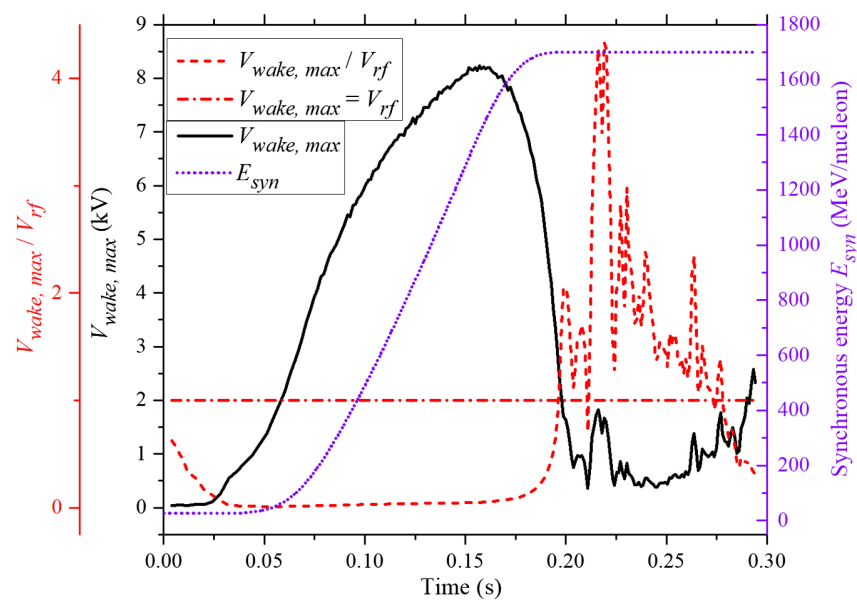

FIG. 5. The maximum wake voltage $\left(V_{\text {wake,max }}\right)$ and the ratio of the maximum wake voltage to the programmed rf voltage of the ${ }^{78} \mathrm{Kr}^{19+}$ beam during a whole operation process. The maximum wake voltage increases to several times of the programmed $\mathrm{rf}$ voltage during the debunching stage, which indicates a drastically distorted bucket. 


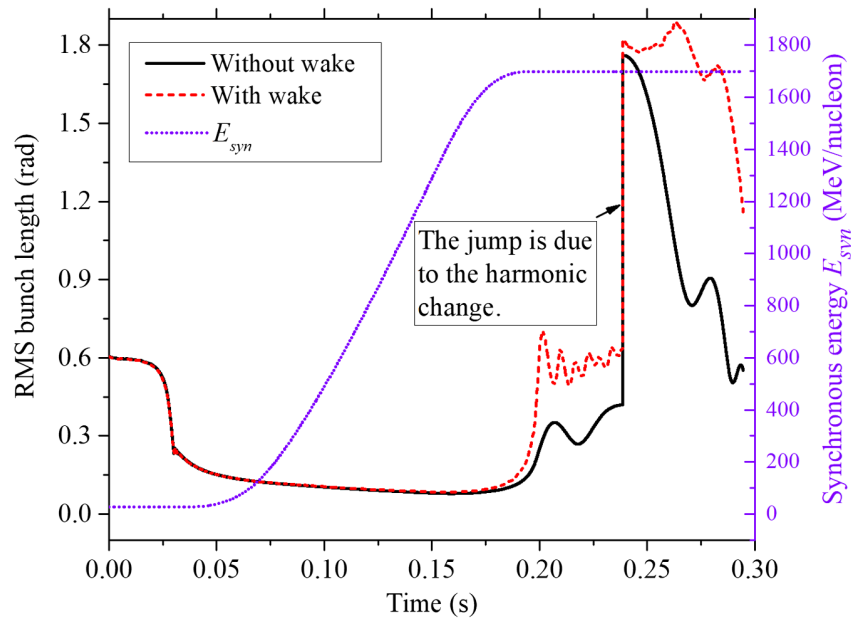

FIG. 6. RMS bunch length of the ${ }^{78} \mathrm{Kr}^{19+}$ beam. The beam is not bunched well at the end of the acceleration stage. At the extraction time, the bunch length increases by $119 \%$ in the presence of wake.

programmed rf voltage is larger than $200 \mathrm{kV}$. Since the wake voltage is small compared to the programmed $\mathrm{rf}$ voltage, the influence of the wake voltage on the beam is small. Then the bucket height decreases, which leads to longer bunches. Thus the maximum wake voltage decreases. However, since the wake voltage decreases more slowly than the programmed rf voltage, the influence of the wake voltage becomes larger and larger. After the acceleration, the programmed rf voltage slowly decreases to its minimum value while the wake voltage increases to several times of the programmed rf voltage. Thus the bucket will be distorted drastically, which can result in the longitudinal emittance growth of the beam.

The RMS bunch length $\left(L_{\mathrm{rms}}\right)$ of the ${ }^{78} \mathrm{Kr}^{19+}$ beam is shown in Fig. 6. The difference between the bunch length with and without wake voltage is small during the first capture stage due to the small wake voltage. The difference is also small during the acceleration stage due to the small ratio of the wake voltage to the programmed rf voltage. However, at the debunching stage and the second capture stage, the bunch length has obvious growth in the presence of wake. At the extraction time, the bunch length increases by $119 \%$, which means that the beam is not bunched well. The jump of RMS bunch length is due to rebunching with a different rf harmonic number.

The RMS momentum spread $\left(\delta_{\text {rms }}\right)$ of the ${ }^{78} \mathrm{Kr}^{19+}$ beam is shown in Fig. 7. The influence of wake on the momentum spread is similar to the influence on the bunch length at each stage. At the extraction time, the RMS momentum spread increases by $111 \%$.

Both the bunch length and the momentum spread increase by more than $100 \%$ in the presence of wake, which means the bucket is severely distorted.

Since the bunch will be extracted to Spectrometer Ring (SRing), the bunch length should be shorter than the

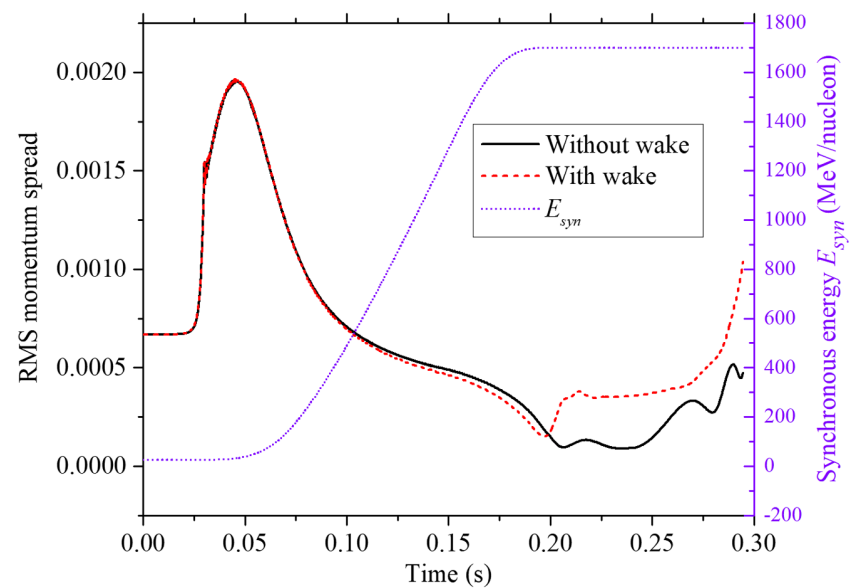

FIG. 7. RMS momentum spread of the ${ }^{78} \mathrm{Kr}^{19+}$ beam. The momentum spread, which has obvious growth at the debunching stage and the second capture stage, increases by $119 \%$ at the extraction time in the presence of wake.

flat-top length $\left(l_{\mathrm{kk}}\right)$ of the injection kicker of the SRing to obtain high transfer efficiency. The maximum $l_{\mathrm{kk}}$ can be defined as

$$
l_{\mathrm{kk}, \max }=\frac{T_{0, \mathrm{SR}}-T_{\mathrm{fall}}}{T_{0, \mathrm{SR}}} \cdot C_{\mathrm{SR}}
$$

where $T_{0, \mathrm{SR}}$ is the revolution period of the beam in the SRing, $T_{\text {fall }}$ is the fall time of the injection kicker of the SRing, and $C_{\mathrm{SR}}$ is the circumference of the SRing. The ideal transfer efficiency $\left(\eta_{\text {trans }}\right)$, which is defined as the percent of the particles that are located within $l_{\mathrm{kk}, \max } / 2$ from the bunch center, is used to measure the severity of beam loading effects in the BRing. According to the simulation results, the ideal transfer efficiency of the ${ }^{78} \mathrm{Kr}^{19+}$ beam decreases to $78.2 \%$ in the presence of wake which is far less than the desired efficiency larger than $99.9 \%$.

Because the beam emittance and the transfer efficiency cannot meet the requirements of the BRing in the presence of wake, the transient beam loading effects should be compensated. The fast feedback system, which is considered as a powerful system for transient beam loading compensation, can not be employed in the BRing because the required loop delay is too small with the small Q value [17]. A multiharmonic feed-forward system is proposed to compensate the beam loading effects in the BRing. A similar feed-forward system is employed in J-PARC RCS and works well for the compensation of transient beam loading effects [18]. In a multiharmonic feed-forward system, the beam signals are picked up by a wall current monitor (WCM). The WCM signals are digitized and then sent to I/Q detection blocks for different harmonics. The compensation signals are obtained from these harmonics separately, summed up and then sent to the amplifier of the cavity. 


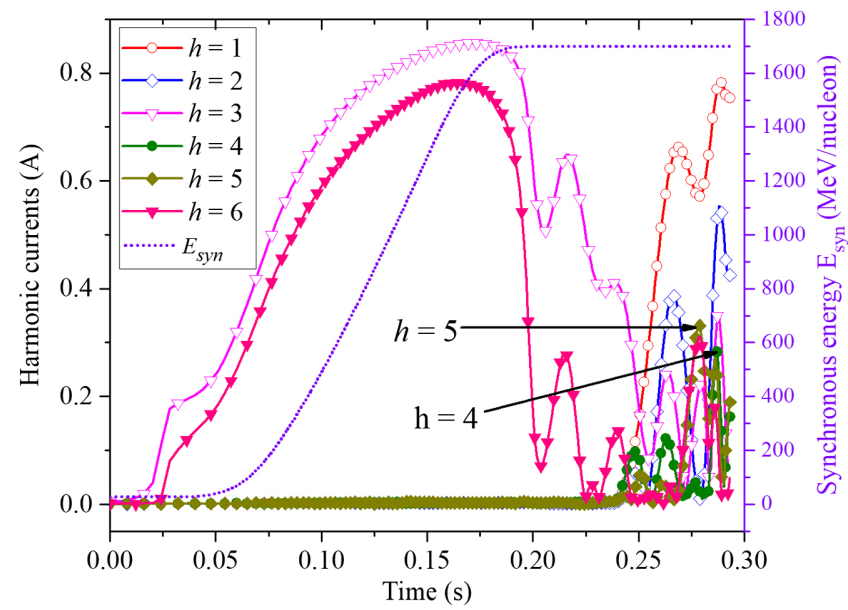

FIG. 8. Harmonic currents of the ${ }^{78} \mathrm{Kr}^{19+}$ beam. Harmonics at $h=1,2,4$, and 5 begin to increase since the second capture stage starts.

The compensation of the wake voltage during the debunching stage and the second capture stage is considered first, because the beam loading effects have large influence on the beam during these two stages according to the simulation results. When the rf harmonic number changes from 3 to 1 , many harmonic currents of the beam can exist. The harmonic currents at $h=1,2,3$, and 6 are important because they are large. Other harmonic currents, especially at $h=4$ and 5, increase a lot during the second capture stage as shown in Fig. 8. When the beam is accelerated to the extraction energy, the frequencies of the harmonic currents at $h=4$ and 5 are close to the resonance frequency of the cavities as shown in Fig. 1. Hence these two harmonic currents should not be ignored. Harmonic currents at $h>6$ should have small influence on the beam because they are either small or far from the resonance frequency of the cavities.

The simulations of compensation are performed with different scenarios, in which the harmonic currents to be compensated are added one by one until the ideal transfer efficiency is close to the case without wake voltage, namely larger than $99.9 \%$. Since the harmonic currents cannot be compensated completely in the operation of an accelerator, the compensation efficiency is set at $90 \%$ in the simulations according to the commissioning results of J-PARC RCS [18]. Since there is a delay time during the operation of the feed-forward system, including the group delay of the system and the delay of signals from the WCM to the position of the system, the beam distribution 3 turns (larger than $6 \mu \mathrm{s}$ ) earlier is picked up with a sampling frequency of $256 \mathrm{MHz}$.

The simulation results also support the analysis of which harmonic currents should be compensated.

The ideal transfer efficiency is used to measure if the compensation is satisfactory. The results of the ideal transfer efficiency with wake voltage compensated are

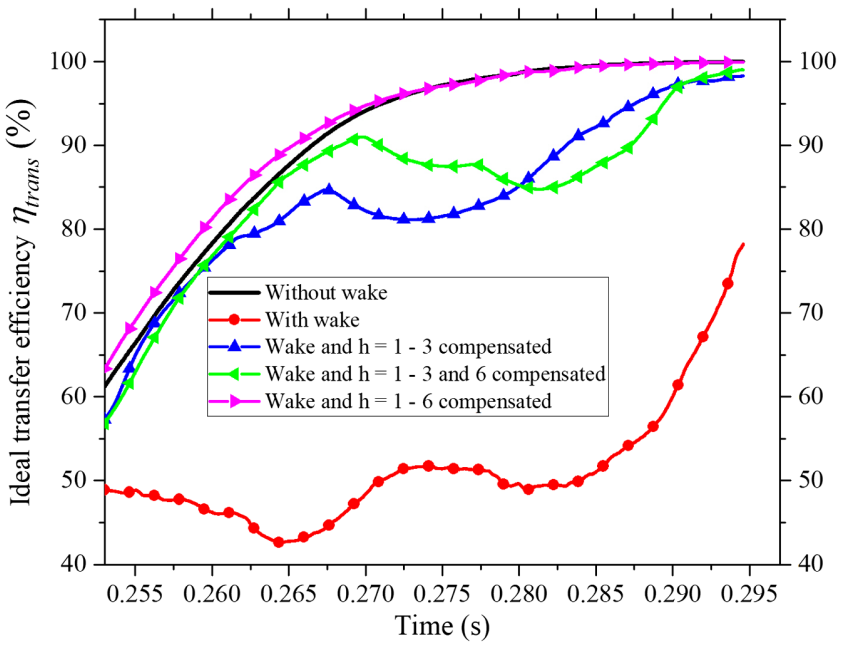

FIG. 9. Ideal extraction efficiency of the ${ }^{78} \mathrm{Kr}^{19+}$ beam with wake compensation. The efficiency reaches $100 \%$ with harmonic currents at $h=1-6$ compensated.

shown in Fig. 9. Though the ideal transfer efficiency should be defined only at the extraction time, it is also shown at the second capture stage to help analyze the influence of each harmonic current. If the harmonic currents at $h=4$ and 5 are not compensated, the efficiency starts to decrease at time $0.27 \mathrm{~s}$ when the two harmonic currents start to increase as shown in Fig. 8. Thus the compensation of these two harmonic currents is necessary.

Table III lists the simulation results at the extraction time with different harmonic currents compensated during the last two stages, namely the debunching stage and the second capture stage. With 6 harmonic currents compensated, both the RMS bunch length and the RMS momentum spread are close to that without wake, and the ideal transfer efficiency reaches $100 \%$, which means the beam loading effects are compensated effectively.

It is of importance to estimate the needed power and the bandwidth of the feed-forward system.

The needed power is related to the wake voltage that should be compensated. The largest wake voltage reaches $8.2 \mathrm{kV}$, and the largest programmed rf voltage is $240 \mathrm{kV}$. So the power supplied to the rf system should be increased by $7 \%$ to compensate the wake voltage. Since the largest

TABLE III. Parameters of the beam at the extraction time.

\begin{tabular}{|c|c|c|c|c|}
\hline \multicolumn{2}{|l|}{ Manipulation } & $\begin{array}{l}L_{\text {rms }} \\
(\mathrm{rad})\end{array}$ & $\begin{array}{c}\delta_{\mathrm{rms}} \\
\left(\times 10^{-4}\right)\end{array}$ & $\begin{array}{c}\eta_{\text {trans }} \\
(\%)\end{array}$ \\
\hline \multicolumn{2}{|c|}{ Without wake } & 0.55 & 4.75 & 100.0 \\
\hline \multicolumn{2}{|c|}{ With wake } & 1.16 & 10.4 & 78.2 \\
\hline Compensated & $h=1-3$ & 0.67 & 6.10 & 98.3 \\
\hline harmonic & $h=1-4$ & 0.62 & 5.10 & 99.5 \\
\hline currents during & $h=1-5$ & 0.58 & 4.93 & 100.0 \\
\hline the last & $h=1-6$ & 0.55 & 4.78 & 100.0 \\
\hline two stages & $h=1,2,3,6$ & 0.63 & 6.01 & 99.0 \\
\hline
\end{tabular}


wake voltage appears at the end of the acceleration with the maximum $\dot{B}$, where the programmed rf voltage has already decreased from $240 \mathrm{kV}$ to about $200 \mathrm{kV}$, the extra power, which is supplied to the rf system for the compensation, should be less than $7 \%$.

The bandwidth of the feed-forward system depends on the harmonic currents that should be compensated and the longitudinal impedance of the rf cavities. To compensate the beam loading effects of all ion beams in the BRing in a whole operation process, the lowest frequency of the band corresponds to ion beams with the lowest $h f_{0}$, and the highest frequency of the band corresponds to ion beams with the highest $h f_{0}$. As shown in Fig. 1, the lowest frequency of the band depends on the ${ }^{238} \mathrm{U}^{35+}$ beam with the lowest revolution frequency of $0.099 \mathrm{MHz}$ at injection and harmonic of 3 (Harmonic currents at $h=1$ and 2 are small as shown in Fig. 8). Thus the lowest frequency of the band should be less than $0.297 \mathrm{MHz}$. The highest frequency of the band depends on the ${ }^{12} \mathrm{C}^{6+}$ beam with the largest revolution frequency of $0.52 \mathrm{MHz}$ and harmonic of 6 . Thus the highest frequency of the band should be larger than $3.12 \mathrm{MHz}$. The feed-forward system should cover frequencies of $0.297-3.12 \mathrm{MHz}$. To separate different harmonic currents at the beginning of the acceleration, the lowest passband of the wave filter of the feedforward system should be less than $0.297 \mathrm{MHz}$.

\section{B. Microwave instability of coasting beams}

The momentum spread in the presence of wake quickly increases during the debunching stage (at about $0.2 \mathrm{~s}$ ) as shown in Fig. 7, which indicates an instability of the coasting beam. In the slow extraction mode, the bunches are debunched as a coasting beam at the end of the acceleration stage. Since the slow extraction can last several seconds, minutes or even longer, it is essential to ensure the stability of the coasting beam.

For a beam below transition with Gaussian distribution in momentum spread, the growth contours, which are derived from the dispersion relation [11], are shown in Fig. 10. The dimensionless coordinates $(U, V)$ are defined by

$$
U+i V=\frac{D e I_{0}\left(Z\left(h \omega_{0}\right) / h\right)}{|\eta| \beta^{2} E \delta^{2}},
$$

where $I_{0}$ is the average current of the beam. The solid line is the stability threshold, and the area outside the line is unstable. Each dash line refers to the same growth rate. From inside to outside, the growth rate increases in steps of $51 \mathrm{~s}^{-1}$ with the RMS momentum spread set at 0.00013 according to the simulation results. The coordinates $(U, V)$ of the coasting ${ }^{78} \mathrm{Kr}^{19+}$ beam for the slow extraction are calculated with Eq. (16) and plotted in Fig. 10. It can be seen that the modes at $h=1-6$ are in the unstable area. When the harmonic number increases, the growth rate

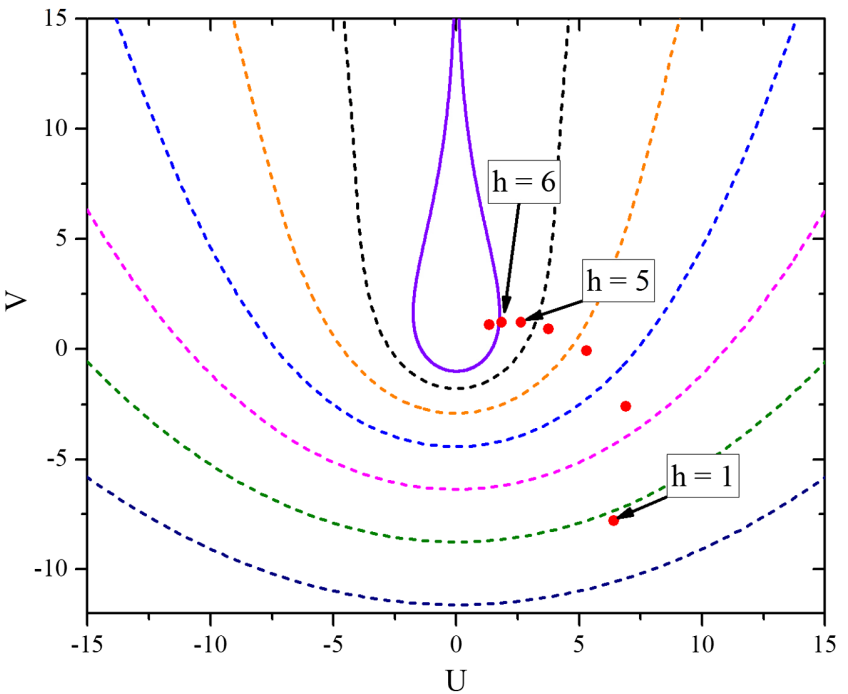

FIG. 10. Growth contours for coasting beams with Gaussian distribution in momentum spread. The solid line is the stability threshold, and the area outside the line is unstable. Each dash line refers to the same growth rate. From inside to outside, the growth rate increases in steps of $51 \mathrm{~s}^{-1}$ with the RMS momentum spread set at 0.00013 . The coordinates $(U, V)$ of the coasting ${ }^{78} \mathrm{Kr}^{19+}$ beam for the slow extraction are calculated with Eq. (16) and plotted in the figure.

decreases. The modes at $h>6$ are in the stable area. The Keil-Schnell criterion [19]

$$
\frac{D e I_{0}\left|Z\left(h \omega_{0}\right) / h\right|}{\Gamma|\eta| \beta^{2} E}<\delta^{2}
$$

can be used to approximate the stability threshold of coasting beams where $\Gamma \approx 1$ for Gaussian distribution. According to the Keil-Schnell criterion, the modes at $h=$ 1-7 are unstable, and the modes at $h>7$ are stable.

Since the slow extraction can last a rather long time, it is not feasible to determine the largest tolerable growth time of the instability. So it is the threshold rather than the growth time that should be cared more about. To find the momentum spread threshold $\left(\delta_{\mathrm{th}}\right)$ of the microwave instability of coasting beams, more simulations are performed. The density distribution of the beams in the longitudinal phase space is assumed to be uniform on the azimuth angle axis and Gaussian on the momentum spread axis. To reduce the impact of numerical noise, up to $10^{6}$ macroparticles are tracked.

The development of self-bunching, which results in the exponential growth of momentum spread of the coasting ${ }^{78} \mathrm{Kr}^{19+}$ beam with initial RMS momentum spread of 0.00013 , is shown in Fig. 11. The coasting beam is severely bunched in less than $40 \mathrm{~ms}$ (about 20000 turns). For different initial momentum spread $\left(\delta_{i}\right)$, the final momentum spread $\left(\delta_{f}\right)$ is shown in Fig. 12. To keep the coasting ${ }^{78} \mathrm{Kr}^{19+}$ beam stable, all the modes should be stable. 


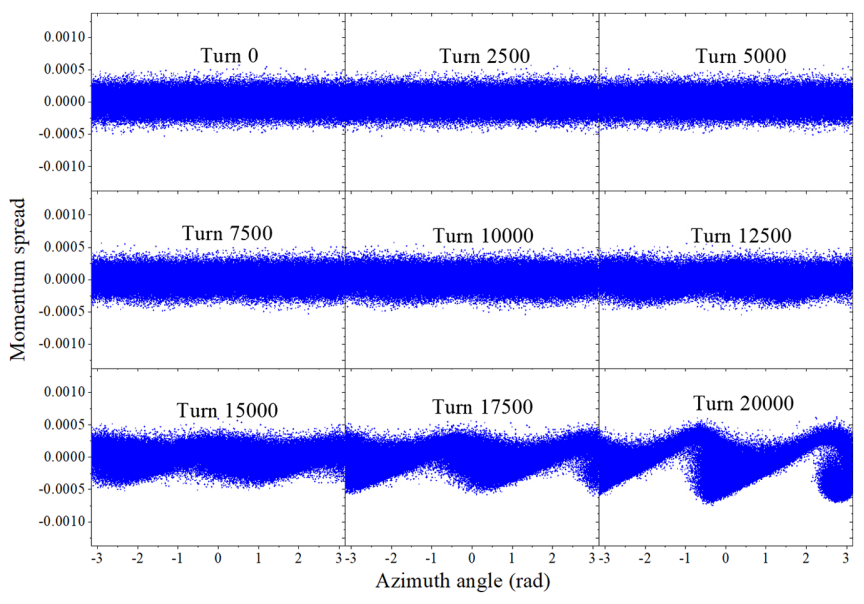

FIG. 11. Self-bunching development of the coasting ${ }^{78} \mathrm{Kr}^{19+}$ beam. The initial beam distribution is uniform on the azimuth angle axis and Gaussian on the momentum spread axis. The initial RMS momentum spread is set at 0.00013 .

The thresholds given by the Keil-Schnell criterion and the growth contours are also shown. The final momentum spread is always larger than the threshold given by the growth contours. If the initial momentum spread is smaller than the threshold, the momentum spread will increase and cross the threshold, and there will be an overshoot [20]. The threshold of RMS momentum spread of the coasting ${ }^{78} \mathrm{Kr}^{19+}$ beam with designed beam intensity and extraction energy is 0.00039 . The simulation results agree with the theoretical analysis.

According to the overshoot formulas $[20,21]$

$$
\delta_{i} \cdot \delta_{f} \approx \delta_{\mathrm{th}}^{2} \quad \text { or } \quad \delta_{i}^{2}+\delta_{f}^{2} \approx \delta_{\mathrm{th}}^{2}
$$

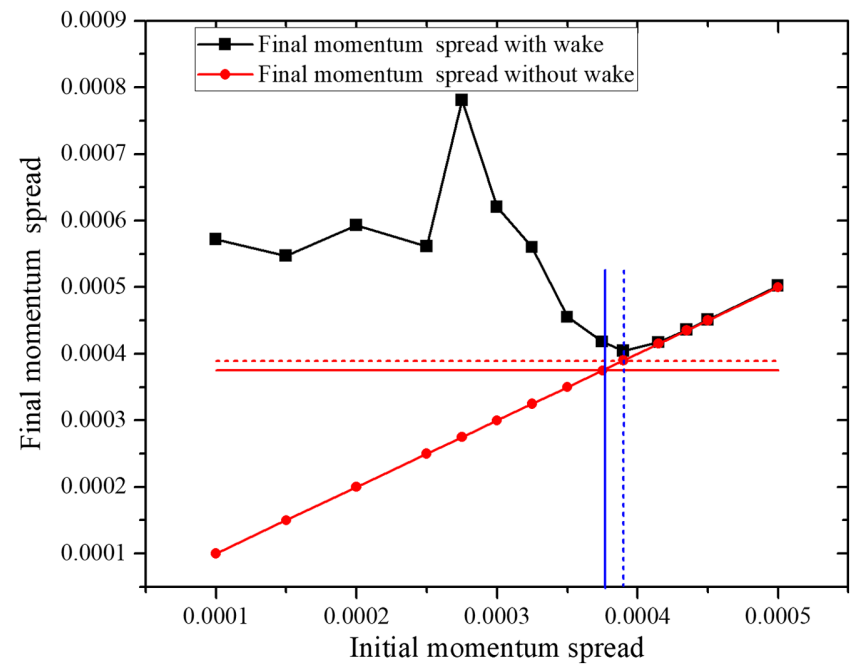

FIG. 12. The RMS momentum spread and stability threshold of the coasting ${ }^{78} \mathrm{Kr}^{19+}$ beam. The solid line is the threshold given by the growth contours, and the dash line is the threshold given by the Keil-Schnell criterion. a smaller initial momentum spread will result in a larger final momentum spread. However, in the simulations, the initial momentum spread of 0.000275 corresponds to the largest final momentum spread. As shown in Fig. 13, the microwave instabilities are driven by the harmonic current at $h=2$ if the initial momentum spread is smaller than 0.00025. During the development of selfbunching, the wake voltage bunches the beam just as a rf voltage does. The wake voltage has many harmonic components, and some of the components will create buckets. The self-bunching is most probably driven by the harmonic component which creates a bucket with the largest height or area [11]. Since the height and the area of the bucket created by the harmonic component at $h=2$ are the largest, the instabilities are driven by the harmonic current at $h=2$. With the initial momentum spread increasing, the growth rates of the instabilities driven by the harmonic current at $h=2$ decrease. When the initial momentum spread is around 0.00025 , the harmonic current at $h=2$ still drives the instabilities together with the harmonic current at $h=1$. If the initial momentum spread is larger than 0.00025 , the instabilities are driven by the harmonic current at $h=1$. This is why the trend of the final momentum spread in the presence of wake changes a lot when the initial momentum spread is smaller than 0.000275 . It might be the reason why the simulation results and experiment results do not agree well with the overshoot formulas when the initial energy spread is too small compared to the threshold [20,21].

Coasting beams also exist before the first capture stage during which the momentum spread of the coasting beams is large, and the beam current is small. Thus the coasting

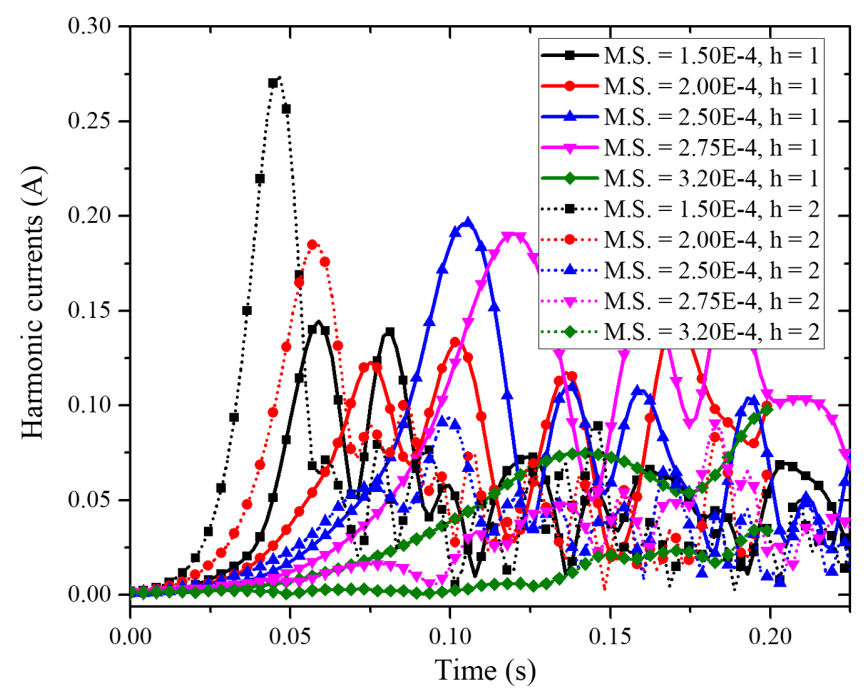

FIG. 13. The harmonic currents at $h=1$ and 2 during the development of instabilities with different initial RMS momentum spread (M.S.). The instabilities are driven by harmonic current at $h=2$ if the initial momentum spread is smaller than 0.00025 and driven by harmonic current at $h=1$ when the initial momentum spread is larger than 0.00025 . 
beams are stable according to simulations and theoretical analysis.

Therefore, in the absence of a feedback system, the RMS momentum spread of the coasting beams for the slow extraction should be larger than the threshold to keep the beams stable. Coasting beams with the largest momentum spread of less than \pm 0.001 are preferred in the BRing, which limits the largest RMS momentum spread to about 0.00033. To obtain stable coasting beams with momentum spread lower than the threshold, there are mainly two methods. One method is to apply a feedback system. A digital feedback system was considered to control the self-bunching of low energy beams in CELSIUS [22,23]. However, such a feedback system is difficult to be applied for the high energy coasting beams in the BRing. The other method is to design a switch to short-circuit the cavity gaps during the slow extraction, which can decrease the impedance of the cavities. Such MA loaded cavities with a shortcircuit switch are under study at IMP.

\section{CONCLUSIONS}

Beam loading effects and the microwave instability of coasting beams are very important in the BRing in which MA loaded cavities with large shunt impedance and low $Q$ value will be employed. A particle tracking code LPTC is developed to simulate the beam loading effects and the longitudinal beam instabilities in the BRing. According to the simulation results, the beam loading effects is severe during the debunching and the second capture stages. Both the bunch length and the momentum spread increase by more than $100 \%$ at the extraction time in the presence of wake. The simulation results indicate that harmonic currents at $h=1-6$ should be compensated. A multiharmonic feed-forward system is proposed to compensate the beam loading effects in the BRing. The bandwidth of the feed-forward system should cover frequencies of 0.297$3.12 \mathrm{MHz}$. The passband of the wave filter of the feedforward system should be less than $0.297 \mathrm{MHz}$. The power supplied to the rf system should be increased by $7 \%$ to compensate the beam loading effects. Microwave instability of the coasting ${ }^{78} \mathrm{Kr}^{19+}$ beam are observed in the simulations. Self-bunching develops and results in the growth of momentum spread if the initial RMS momentum spread is lower than the threshold, namely 0.00039 for the coasting ${ }^{78} \mathrm{Kr}^{19+}$ beam. The microwave instability can be driven by different harmonic components with different initial RMS momentum spread. MA loaded cavity with a switch to short-circuit the cavity gaps is proposed to suppress the microwave instability of coasting beams in the BRing.

\section{ACKNOWLEDGMENTS}

This work is supported by the National Key R\&D Program of China (Grant No. 2019YFA0405400),
National Natural Science Foundation of China (Grant No. 11825505), and Guangdong Innovative and Entrepreneurial Research Team Program (Grant No. 2016ZT06G373). Many thanks to Y. S. Yuan for useful comments and suggestions.

[1] J. C. Yang, J. W. Xia, G. Q. Xiao et al., High intensity heavy ion accelerator facility (HIAF) in China, Nucl. Instrum. Methods Phys. Res., Sect. B 317, 263 (2013).

[2] G. F. Qu, W. P. Chai, J. W. Xia et al., Two-plane painting injection scheme for BRing of HIAF, Nucl. Sci. Tech. 28, 114 (2017).

[3] S. Ruan, J. C. Yang, J. Q. Zhang et al., Design of extraction system in BRing at HIAF, Nucl. Instrum. Methods Phys. Res., Sect. A 892, 53 (2018).

[4] Y. Mori, M. Fujieda, K. Koba et al., A new type of rf cavity for high intensity proton synchro-tron using high permeability magnetic alloy, in Proceedings of 6th European Particle Accelerator Conference (EPAC 98) (1998), https://lib-extopc.kek.jp/preprints/PDF/1998/9827/ 9827103.pdf

[5] C. Ohmori, S. Anami, E. Ezura et al., High field gradient cavity for J-PARC $3 \mathrm{GeV}$ RCS, in Proceedings of the 9th European Particle Accelerator Conference, Lucerne, 2004 (EPS-AG, Lucerne, 2004), p. 123 [http://accelconf .web.cern.ch/AccelConf/e04/].

[6] C. Ohmori, O. Araoka, E. Ezura et al., High gradient magnetic alloy cavities for J-PARC upgrade, in Proceedings of the 2nd International Particle Accelerator Conference, San Sebastiáán, Spain (EPS-AG, Spain, 2011), Vol. 2885.

[7] F. Tamura, C. Ohmori, K. Hasegawa et al., Beam test of the CERN PSB wide-band rf system prototype in the J-PARC MR, in Proceedings of IPAC2014, Dresden, Germany (JACoW Publishing, Geneva, Switzerland, 2014), https:// doi.org/10.18429/JACoW-IPAC2014-THPME065.

[8] U. Laier, G. Hülsmann, K. P. Ningel, and G. Schreiber, Design of an MA based rf system for the collector ring at FAIR, Particle Accelerator Conference (PAC 09) (JACoW, Geneva, Switzerland, 2010), TU5PFP024.

[9] M. Fujieda, Y. Iwashita, A. Noda et al., Magnetic alloy loaded rf cavity for barrier bucket experiment at the AGS, in Proceedings of the 18th Particle Accelerator Conference, New York, 1999 (IEEE, New York, 1999), Vol. 2, pp. 857-859.

[10] M. Yamamoto, M. Fujieda, Y. Hashimoto et al., Beam loading effects on high gradient MA-loaded cavity, in Proceedings of the 18th Particle Accelerator Conference, New York, 1999 (IEEE, New York, 1999), Vol. 2, pp. 860-862.

[11] K. Y. Ng, Physics of Intensity Dependent Beam Instabilities (World Scientific, Singapore, 2006).

[12] J. A. MacLachlan, Multiparticle dynamics in the e- $\varphi$ tracking code esme, AIP Conf Proc. No. 642, 68 (2002).

[13] CERN Beam Longitudinal Dynamics Code BLonD, http:// blond.web.cern.ch.

[14] A. W. Chao, Physics of Collective Beam Instabilities in High Energy Accelerators (Wiley, New York, 1993). 
[15] P. B. Wilson, Introduction to wakefields and wake potentials, AIP Conf. Proc. No. 184, 525 (1989).

[16] H. Timko, D. Quartullo, A. Lasheen et al., Benchmarking the beam longitudinal dynamics code BLonD, in Proceedings of IPAC 2016, Busan, Korea (JACoW, Geneva, Switzerland, 2016), page WEPOY045, https:// doi.org/10.18429/JACoW-IPAC2016-WEPOY045.

[17] D. Boussard, Design of a ring rf system, in CERN Accelerator School: 4th General Accelerator Physics Course (CERN, Geneva, 1991), p. 294-322.

[18] F. Tamura, M. Yamamoto, C. Ohmori, A. Schnase, M. Yoshii, M. Nomura, M. Toda, T. Shimada, K. Hara, and K. Hasegawa, Multiharmonic rf feedforward system for beam loading compensation in wide-band cavities of a rapid cycling synchrotron, Phys. Rev. Accel. Beams 14, 051004 (2011).
[19] E. Keil and W. Schnell, Concerning longitudinal stability in the ISR, Technical Report No. CERN-ISR-TH-RF-6948, CERN, 1969.

[20] Y. Chin and K. Yokoya, Analytical approach to the overshoot phenomenon for a coasting beam in particle accelerators, Phys. Rev. D 28, 2141 (1983).

[21] R. A. Dory, Nonlinear azimuthal space charge effects in particle accelerators, Doctor of Philosophy (Physics) thesis, University of Wiscosin, 1962.

[22] V. Ziemann, Design considerations for a digital feedback system to control self-bunching in ion-storage rings, Phys. Rev. ST Accel. Beams 4, 042801 (2001).

[23] V. Ziemann, Controlling self-bunching in ion-storage rings, AIP Conf. No. 773, 355 (2005). 